\title{
In-source photocatalytic reduction of disulfide bonds during laser desorption ionization $\dagger$
}

\author{
Liang Qiao, ${ }^{a}$ Hongyan Bi, ${ }^{b}$ Jean-Marc Busnel, ${ }^{b}$ Baohong Liu*a ${ }^{* a}$ and Hubert H. Girault $* b$ \\ Received (in Cambridge, UK) 31st July 2008, Accepted 30th September 2008 \\ First published as an Advance Article on the web 30th October 2008 \\ DOI: $10.1039 / \mathbf{b 8 1 3 2 8 3 f}$
}

A photosensitive plate based on sintered $\mathrm{TiO}_{2}$ nanoparticles has been developed to carry out in-source photo-induced reductions for cleavage of disulfide bridges using glucose as a hole scavenger during laser desorption ionization.

Matrix Assisted Laser Desorption Ionisation (MALDI) is a widely used technique in the growing field of proteomics. ${ }^{1}$ During the ionization step, many complex charge and energy transfer processes inducing photochemical redox reactions take place within a few nanoseconds in the plume, ${ }^{2-4}$ leading to potential useful in-source reactive applications. Organic acids such as sinapinic acid, 2,5-dihydroxybenzoic acid or $\alpha$-cyano-4hydroxycinnamic acid are generally used as matrices, while novel inorganic materials such as $\mathrm{TiO}_{2}$ have been introduced recently either to present additional functions on the target plate or to enhance the ion yield for non volatile analytes. ${ }^{5,6}$ $\mathrm{TiO}_{2}$ nanoparticles can act as sensitizers for light-induced redox processes due to their electronic structure characterized by a filled valence band and an accessible empty conduction band. ${ }^{7}$ Upon irradiation at appropriate wavelengths, electrons are excited from the valence band to the conduction band, yielding electron-hole pairs. ${ }^{7}$ These excited electrons can either be used directly to generate electricity as in photovoltaic solar cells, ${ }^{8}$ or to drive a chemical reaction as in photocatalysis. ${ }^{9}$ This photochemistry approach has been applied to many fields, ${ }^{10-13}$ but not yet widely used in biological sciences. Recently, we have developed a $\mathrm{TiO}_{2}$-based photo-electrode on a target plate to control photo-electrochemical redox reactions during the matrix assisted laser desorption ionization process, where the generated products can be directly analyzed by the time-offlight mass spectrometer. ${ }^{14}$ As a first application, we reported a MALDI in-source photocatalytic oxidation reaction for online peptides tagging. ${ }^{14}$

Herein, we propose another application to drive in-source photo-reduction reactions. As a matter of fact, photocatalytic reductions are much less frequently studied than oxidations, probably because the reducing ability of a conduction band electron is considerably lower than the oxidizing one of a valence band hole. ${ }^{15}$ As reported, the trapping of conduction band electrons on the nanoparticles is very fast. ${ }^{16}$ To prevent

\footnotetext{
${ }^{a}$ Department of Chemistry and Institute of Biomedical Sciences, Fudan University, Shanghai, 200433, China.

E-mail: bhliu@fudan.edu.cn; Fax: +862165641740;

Tel: +862165642405

${ }^{b}$ Laboratoire d'Electrochimie Physique et Analytique, Ecole Polytechnique Fédérale de Lausanne, Station, 6, CH-1015 Lausanne, Switzerland.E-mail:Hubert.Girault@epfl.ch;

Fax: + 4121 6933667; Tel: +41216933145

$\dagger$ Electronic supplementary information (ESI) available: Experimental information. See DOI: 10.1039/b813283f
}

this kind of electron quenching from on-surface or in-volume recombination, it is anticipated that the addition of an electron donor can theoretically be beneficial by scavenging valence band holes and thereby freeing more reductive equivalents. ${ }^{15}$ To date, the photocatalytic reduction of carbon tetrachloride or nitroaromatics in solution has been reported in the presence of a large excess of electron donors, such as isopropanol. ${ }^{17}$ However, there is no example of in-source photocatalytic reduction realized during laser desorption ionization (LDI) using inorganic nanoparticles.

In principle, this photo-reduction concept could be used to carry out in-source protein decay and disulfide bond cleavage, which are typical reduction processes employed in proteomics. ${ }^{18,19}$ Indeed, disulfide bond formation is a post-translational modification that strongly stabilizes the $3 \mathrm{D}$ structure of proteins, and their cleavages is necessary for the rapid sequencing of proteins containing such modifications. ${ }^{18}$ Additionally, disulfide bridge mapping can be an important step for the structural characterization of proteins. The traditional disulfide profiling approaches require several complex pre-treatment strategies such as digestion, chemical reduction, modification and fractionation. ${ }^{20}$ Recently, Tanaka et al. reported a new matrix, 1,5-diaminonaphthalene, to help disulfide reduction for on-line disulfide mapping. ${ }^{20}$ Herein, an alternative protocol based on a photo-catalytic reaction using a $\mathrm{TiO}_{2}$-modified plate is developed to realize the in-source cleavage of disulfide bonds as well as laser desorption ionization with the assistance of hole scavengers.

The $\mathrm{TiO}_{2}$ spot array is made by depositing and sintering P25 $\mathrm{TiO}_{2}$ nanoparticles on a commercial steel target plate for a Bruker Microflex (ESI). To achieve highly effective reduction on the nanoporous $\mathrm{TiO}_{2}$ structure, glucose was used as a nonvolatile electron donor and deposited together with sample proteins on the modified plate. Considering the abundant hydroxyl groups in the carbohydrate, glucose is acting as a strong hole scavenger for the photocatalytic reduction occurring in the ion source. Indeed, it has been proven that glucose can scavenge holes in aqueous media efficiently by using a nanosecond time-resolved transient absorption spectroscopy, where the decay kinetics of electron would flatten out with the presence of the electron donor. ${ }^{21}$ As illustrated in Scheme 1a, under the irradiation by the laser source, the $\mathrm{TiO}_{2}$ nanoparticles can absorb photons generating electron-hole pairs and acting as photosensitisers, where an electron would be injected into the valence band from glucose generating an oxidized glucose, and the excited conduction band electron can therefore be employed to induce the disulfide bond cleavage. The major advantage of the present approach stems from the large specific surface area of the mesoporous structure offering a 
a)

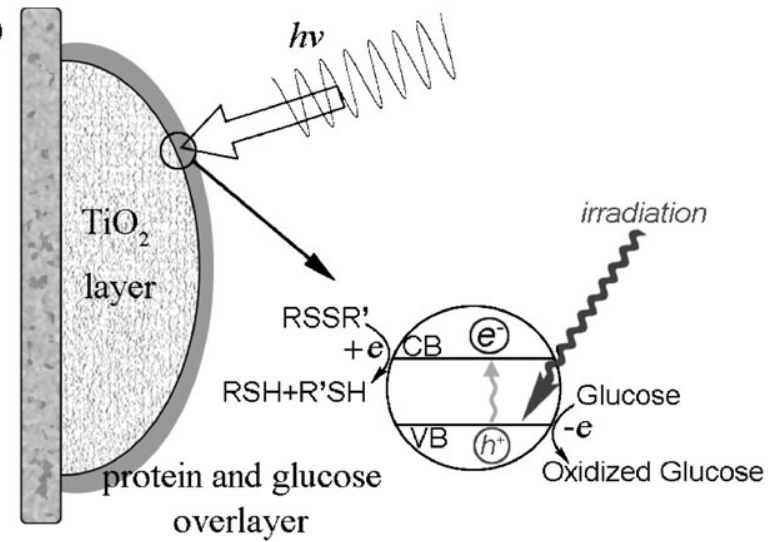

b)

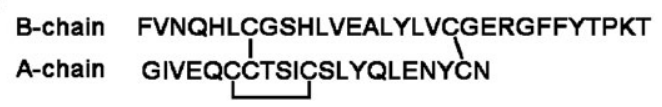

Scheme 1 (a) Schematic representation of the in-source photocatalytic reduction of disulfide bond with the assistance of glucose on a $\mathrm{TiO}_{2}$ photoelectrode plate; (b) Structure of human insulin.

high redox capacity while enabling the very effective determination of the reduction products of a given molecule by mass spectrometry.

To illustrate the present photo-reductive protocol, human insulin was selected as an example to carry out in-source reduction of disulfide bonds during laser desorption ionization. Human insulin is a protein consisting of two peptide chains, i.e. A- and B-chains, and three disulfide bonds, including two bridge bonds and an intra-bond, as shown in Scheme $1 \mathrm{~b}$. By reducing these disulfide bonds, two fragments derived from A- and B-chains can be generated. To study the $\mathrm{TiO}_{2}$ photo-reductive property, the mass spectrometry analysis was first carried out classically in the presence of an organic matrix and then with the $\mathrm{TiO}_{2}$-modified target plate. Fig. $1 \mathrm{~b}$ shows the MALDI mass spectrum of human insulin obtained on a stainless steel target plate with the conventional matrix, DHB, in positive linear mode. The detected ions are primarily derived from the intact insulin molecule as singly charged or doubly charged ions. No significant observation of visible A- and B-chains ions indicates that the reduction reaction cannot efficiently take place with the DHB matrix, which is however known to present some reductive properties. ${ }^{22}$ Conversely, when the same experiment was carried out using the $\mathrm{TiO}_{2}$-based LDI-MS with the assistance of glucose in positive linear mode, two intense peaks representing A- and B-chains were newly observed together with the peak standing for intact human insulin, while the doubly charged ion peak disappeared (Fig. 1a). These data demonstrate that the $\mathrm{TiO}_{2}$-modified plate can be employed efficiently for photocatalytic reduction in the presence of sacrificial electron donor reagents.

To verify the effect of glucose as a hole scavenger during the in-source photocatalytic-reduction procedure, citric acid was used instead for comparison. It has been proven that citric acid could act as an efficient proton donor and hole conductor favoring the long distance redox reactions while assisting sample ionization. ${ }^{5,14}$ Fig. 1c shows the mass spectrum obtained with the $\mathrm{TiO}_{2}$-modified plate for a disulfide bond
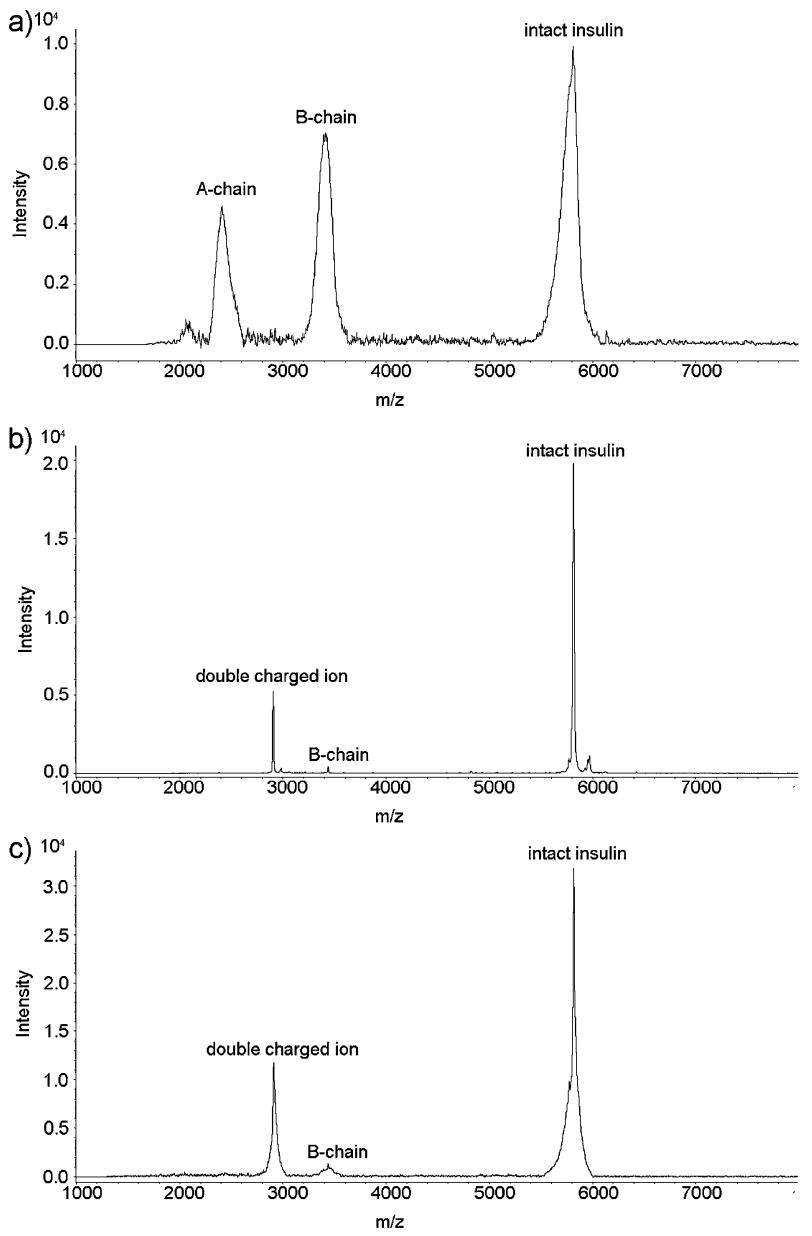

Fig. 1 Mass spectra of human insulin (17 pmol) obtained (a) on the $\mathrm{TiO}_{2}$ photoelectrode plate with the presence of glucose $(10 \mu \mathrm{g})$, (b) on a normal target plate using DHB matrix and (c) on the $\mathrm{TiO}_{2}$ photoelectrode plate with the presence of citric acid $(10 \mu \mathrm{g})$ in linear positive ion mode.

reduction of human insulin in positive linear mode with the assistance of citric acid. In the mass spectrum, only peaks from intact human insulin together with a very weak peak from B-chain are observed, showing that the reductive property is not strong enough using citric acid $\mathrm{TiO}_{2}$-LDI. Without glucose working as a sacrificial hole scavenger, there would be strong volume and surface recombination of electrons and holes, ${ }^{15}$ and thus the number of electrons available for inducing disulfide bonds cleavages would be limited. Therefore, the presence of glucose is essential for enhancing the photo-reductive ability of the system to achieve disulfide bond cleavages.

If the proposed approach appears successful to map the disulfide bonds of insulin, it has to be noticed that the resolution achieved is limited. In the present state, it would be a drawback for practical applications. Different from organic matrices, the sintered $\mathrm{TiO}_{2}$ layer might not supply an ideal environment for ion formation of large molecules. It is generally acknowledged that the incorporation of the analyte into matrix crystals is an important prerequisite for a successful MALDI analysis of proteins and peptides. ${ }^{2,4}$ For the results shown in Fig. 1a and c, it could be hypothesized that 
the analytes are well incorporated into the crystalline glucose or citric acid but separated from the laser energy absorber, i.e. the $\mathrm{TiO}_{2}$ nanoparticles, resulting in a poor mass spectrum, where a spread of the initial velocity of the formed ions occurs. Additionally, based on the cluster model developed by Karas et al., which assumes that the ions are mainly preformed in the solid matrix and then undergo incomplete neutralization in the plume, leaving only some useful ions at the end, ${ }^{2,3}$ it appears realistic that the use of the neutral glucose rather than the acidic matrix induces a smaller formation of ions in the crystal, thus limiting the ionization. The photochemical reactions occurring in the ion source may also induce ion energy spread, which cannot be compensated by the delayed extraction time. Experimental and theoretical investigation leading to a better understanding of the role of the addition of acidic intermediate molecules or redox mediators is now being pursued in order to enhance the ionization ability and catalytic efficiency.

The in-source photo-electrochemical mechanism happening within nano-seconds is a complicated process, where not only final products but also many intermediate active species, such as radicals, electrons and protons, are generated. In this case, it can be proposed that glucose is oxidized by the photoinduced valence band holes giving out electrons and protons, while the disulfide groups of the protein would be reduced by capturing electrons from the conduction band and protons. It is likely that the reduction is initiated either at the surface or in the nanopores of the $\mathrm{TiO}_{2}$ photoelectrode, but that further charge transfer reactions occur in the plume. Benefiting from the large specific surface and pore volume and the confinement effect, the reduction is very effective as being observed. Further investigation of the reduction mechanism is still in progress.

In summary, we have demonstrated that it is possible to perform in-source photocatalytic reduction reactions during laser desorption ionization using a mesoporous $\mathrm{TiO}_{2}$-modified target plate. Specifically, we have carried out the reduction of disulfide bonds in human insulin using glucose as a sacrificial hole scavenger and proton donor. The long term aim of this work is to develop simple in-source cleavage protocols for high throughput on-line sequencing of proteins containing disulfide bonds. More generally, the present system shows a versatile and promising strategy for the rapid study of in-source peptide decay and/or other biomolecular reductions by integrating the sample reduction and ionization in one step followed by mass spectrometry analysis.

This work is supported by NSFC (20775016, 20575013, 20735005) and Swiss-China cooperation research program. BHL is grateful to EPFL for a visiting professor fellowship and $\mathrm{HHG}$ is grateful to Fudan University for a visiting professor fellowship.

\section{Notes and references}

1 R. Aebersold and M. Mann, Nature, 2003, 422, 198-207.

2 M. Karas and R. Kruger, Chemical Reviews, 2003, 103, 427-439.

3 R. Knochenmuss, Analyst, 2006, 131, 966-986.

4 R. Knochenmuss and R. Zenobi, Chemical Reviews, 2003, 103, 441-452.

5 C. T. Chen and Y. C. Chen, Rapid Communications in Mass Spectrometry, 2004, 18, 1956-1964.

6 L. Qiao, C. Roussel, J. J. Wan, P. Y. Yang, H. H. Girault and B. H. Liu, Journal of Proteome Research, 2007, 6, 4763-4769.

7 H. Hennig, Coordination Chemistry Reviews, 1999, 182, 101-123.

8 P. Atienzar, S. Valencia, A. Corma and H. Garcia, Chemphyschem, 2007, 8, 1115-1119.

9 N. Keller, D. Robert, J. M. Herrmann and V. Keller, Catalysis Today, 2007, 122, 1-2.

10 D. Robert, Catalysis Today, 2007, 122, 20-26.

11 D. S. Zhang, J. A. Downing, F. J. Knorr and J. L. McHale, Journal of Physical Chemistry B, 2006, 110, 21890-21898.

12 K. Szacilowski, W. Macyk, A. Drzewiecka-Matuszek, M. Brindell and G. Stochel, Chemical Reviews, 2005, 105, 2647-2694.

13 B. Oregan and M. Gratzel, Nature, 1991, 353, 737-740.

14 L. Qiao, C. Roussel, J. J. Wan, J. Kong, P. Y. Yang, H. H. Girault and B. H. Liu, Angewandte Chemie-International Edition, 2008, 47, 2646-2648.

15 G. Palmisano, V. Augugliaro, M. Pagliaro and L. Palmisano, Chemical Communications, 2007, 3425-3437.

16 G. Rothenberger, J. Moser, M. Gratzel, N. Serpone and D. K. Sharma, Journal of the American Chemical Society, 1985, 107, 8054-8059.

17 J. L. Ferry and W. H. Glaze, Langmuir, 1998, 14, 3551-3555.

18 V. Schnaible, S. Wefing, A. Resemann, D. Suckau, A. Bucker, S. Wolf-Kummeth and D. Hoffmann, Analytical Chemistry, 2002, 74, 4980-4988.

19 M. Takayama and A. Tsugita, International Journal of Mass Spectrometry, 1998, 181, L1-L6.

20 Y. Fukuyama, S. Iwamoto and K. Tanaka, Journal of Mass Spectrometry, 2006, 41, 191-201.

21 I. A. Shkrob, M. C. Sauer and D. Gosztola, Journal of Physical Chemistry B, 2004, 108, 12512-12517.

22 K. Demeure, L. Quinton, V. Gabelica and E. De Pauw, Analytical Chemistry, 2007, 79, 8678-8685. 